\title{
Brute force algorithm implementation for traveljoy travelling recommendation system
}

\author{
K.A.F.A. Samah ${ }^{1}$, N. Sabri ${ }^{2}$, R. Hamzah $^{3}$, R. Roslan ${ }^{4}$, N.A. Mangshor ${ }^{5}$, A.A.M. Asri ${ }^{6}$ \\ 1,2,4,5,6 Faculty of Computer and Mathematical Sciences Universiti Teknologi MARA (Kampus Jasin), Malaysia \\ ${ }^{3}$ Faculty of Computer and Mathematical Sciences, Faculty of Computer and Mathematical Sciences \\ Universiti Teknologi MARA Shah Alam, Malaysia
}

\section{Article Info}

Article history:

Received Jan 9, 2019

Revised Mar 15, 2019

Accepted May 18, 2019

\section{Keywords:}

Optimization place of interest brute force

Recommendation system Traveljoy

\begin{abstract}
This paper presents the Brute Force algorithm implementation for TravelJoy Travelling Recommendation System. Due to overwhelmed information in the internet, travelers faced difficulties in finding and comparing which places in Melaka that worth to visit. Melaka is a well-known place as one of the most popular tourist spots in Malaysia, famous with historical places. All the mentioned problems were time-consuming and required lots of efforts for manual comparison between places and planning the trip itinerary. An efficient application system is needed to assist travelers in planning their trip itinerary by providing details of interesting place in Melaka, budget estimating and recommendation of sequence places which to visit. The TravelJoy application applied Traveling Salesman Problem (TSP) concept using Brute Force algorithm in determining the least time duration for the selected places and adapting Expected Time Arrival (ETA). It was found through Brute Force algorithm adaptation; the recommendation system is reliable based on the functional and reliability testing with t-test result of 0.00067 , indicates the system is accepted.
\end{abstract}

Copyright (C) 2019 Institute of Advanced Engineering and Science. All rights reserved.

Corresponding Author:

K.A.F.A. Samah,

Faculty of Computer and Mathematical Sciences,

Universiti Teknologi MARA (Kampus Jasin),

77300 Merlimau, Melaka, Malaysia.

Email: khyrina783@melaka.uitm.edu.my

\section{INTRODUCTION}

Melaka is one of Malaysia's famous tourist destinations with its cultural heritage and historic places to visit [1], a unique place where everybody never wanted to miss due to its existence as a living heritage city [2] and always been set as a family trip destination [3]. There are many Place of Interest (POI) in Melaka and most of the places are located in close proximity to each other. As the rapidly growing of the Internet, the travel information can be found on many platforms such as blogs, forums and review websites [4]. However, due to this massive information, travelers got overwhelmed [5] and yet could not determine which place of interest worth to visit [6] especially unfamiliar places [7], confused to make a decision and makes them tired out in considering the place of interest, the budget [8] and uses of time and activities schedule [9].

Besides, travelers also found it is time-consuming to study the duration of the trip and the sequence of the selected places which to visit first [7] and have difficulty in estimating their time arrival for each trip. They also went to local office tourist to gain information about the place they are visiting and seeks for the staff helps [10]. According to [11], travelers may face some unpredicted problems and changes of plan, hence they need to be able to get new information for their trip [12]. Travelers depend on the smartphone to know a certain location and they did not know the sequences of the selected places so they get a minimal duration. This supported through a survey towards 127 respondents where $85.8 \%$ agreed. While $98.4 \%$ claimed that they need a system that gives minimal duration to the selected places they want to visit and the way back 
home. This minimal route is a crucial factor for travelers to make traveling time shorter [13] in order to maximize their satisfaction and experience [10]. In addition, 89.8\% agreed that the expenses exceed the planned budget during travel. Considering on-time arrival for the shortest route problem would provide a reliable path for travelers to plan their trip and [14] described that people tend to overspend their money when they unable to control their desires. Therefore, in this study, this optimization system is developed to increase the traveler's satisfaction through the optimal solution for minimize their route, help them to choose the place of interests and keep their budget in control. Furthermore, 96.9\% agreed this system be developed in the mobile platform and as [15] supported that mobile application as the attractive platform as it is a useful device in tourism area due to its size, the capabilities [16] and able to use real-time intelligent recommender system [17]. These technologies can help in planning the trip more efficiently by reducing cost, less timeconsuming and efforts in finding the right information [18]. There are few applications such as iMelaka, Destination Melaka, Malacca Travel Guide, and Jalan-Jalan Malacca which available in the Google Play Store. Among all those existing applications, it provides information such as restaurants, shopping malls, accommodations, and attractions places. Despite the information is the same, yet none of it provides features such as precise information by separating the categories, ETA, budget estimation and the sequence navigation of the selected POI.

A Travelling Salesman Problem (TSP) concept using Brute Force (BF) algorithm is applied in this application in determining the least time duration for the traveler starting from the traveler's current location, to the interesting places and return back to the current location. The current location and the chosen POI act as input for this algorithm implementation. It will help travelers in planning their trip itinerary by providing details of POI in Melaka, calculate their budget, give the recommendation of the sequence of places to visit first with the shortest duration. Furthermore, this application is linked to Global Positioning System (GPS) data and applied Expected Time Arrival (ETA) concept by using Direction Application Program Interface (API) to optimize the result of this mobile application usage. There were 20 places of interest in Melaka Tengah originally provided by the application to the travelers and controlled by the admin. This application is functional for a day trip only in Melaka and the mode of duration is traveling by car. Besides, calculating the budget based on the ticket entrance only as it is the most important part of planning a trip's budget.

An optimization technique is applied in the recommendation system for the tourism industry to enhance the traveler's experience. Finding the shortest route is the main problem to be solved in developing a recommendation system for traveling [19]. Next, the recommender system is a system needed to help the traveler to get the precise result that suits their interest by giving a suggestion or recommendation. As agreed by [20], the recommender system advice user about what decision or action to take especially when users are overwhelmed with too much information. There were only two algorithms for TSP optimization technique, which are the BF algorithm and Branch and Bound (BB) algorithm. Despite BF and BB has the same advantages such as able to find the shortest path more than two cities and able to return to the initial point, yet the BB calculation is too complex and chooses solution at each level without considering next path. BF has more advantage due to less complexity and easy to implement and choose the optimal solution at the end of the calculation of path. Thus, BF algorithm has been chosen due to its efficiency, suitable in finding the shortest path [21], fulfill the concept of TSP and able to find the optimal solution.

Therefore, this paper presents the BF implementation for TravelJoy traveling recommendation system to overcome overwhelming information problem. This paper is organized as follows. In section 1 , we have reviewed some background and literature of the developed system. The methodology is explained in Section 2. The results and discussion are in Section 3 and finally Section 4 for the conclusion of the paper.

\section{RESEARCH METHOD}

In this study, the methodology is using Mobile Application Development Lifecycle (MADLC). It has been chosen due to its best features that contain agile concept which can adapt to changes quickly [22] and suited the development of an application. As stated by [23], the mobile application is no longer a simple system and it needs a new method to handle the mobile application development compared to the traditional life cycle in solving certain constraints such as memory capacity, interface design and processing power [24]. The best part of this methodology is it allows the continuous process between the requirements, development and the testing until the final prototype finished. The important of the methodology is divided into three parts which are use case diagram, TravelJoy system workflow and the implementation of the BF algorithm

\subsection{Use Case Diagram}

A use case diagram illustrates the interaction of traveler and the admin with the traveling recommendation system as in Figure 1. This is important to show the sequence of actions and the interactions 
involved to achieve the objectives. Use case description is important to clearly explain with details each use case involved so the developer understands exactly what is in the requirement to satisfy.

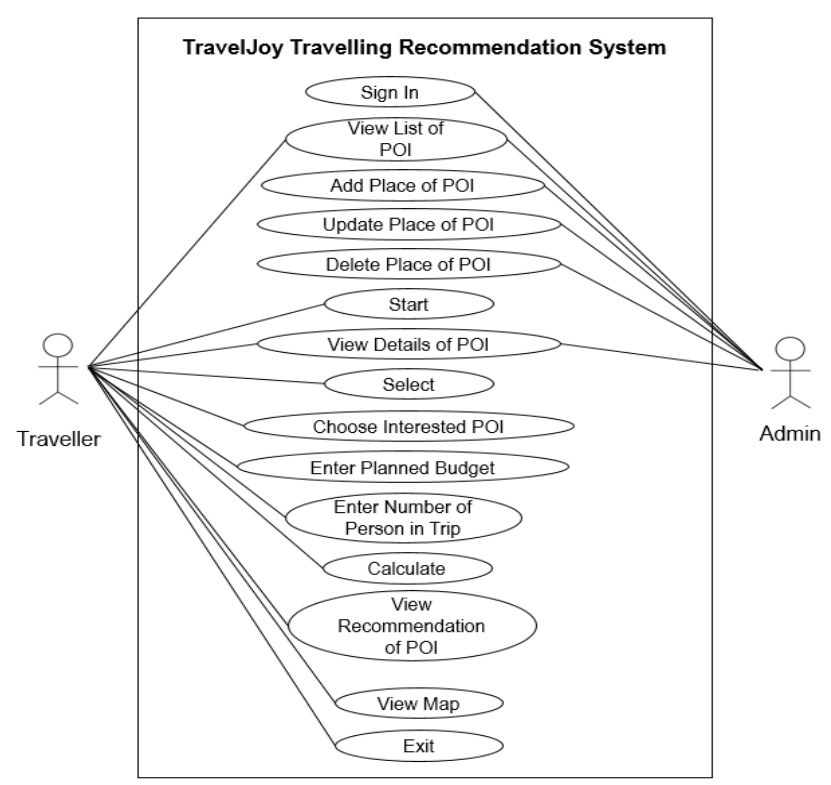

Figure 1. TravelJoy overall system use case diagram

\subsection{Flowchart Diagram}

A flowchart diagram illustrates the sequence of a process in a system using different kind of symbols which contain information. Figure 2 illustrates the flowchart diagram on the exact sequences of the developed TravelJoy recommendation system started with the selection of interested POI by traveler since the system flow is important in designing a system as to avoid any obstacles and the system is clearly described.

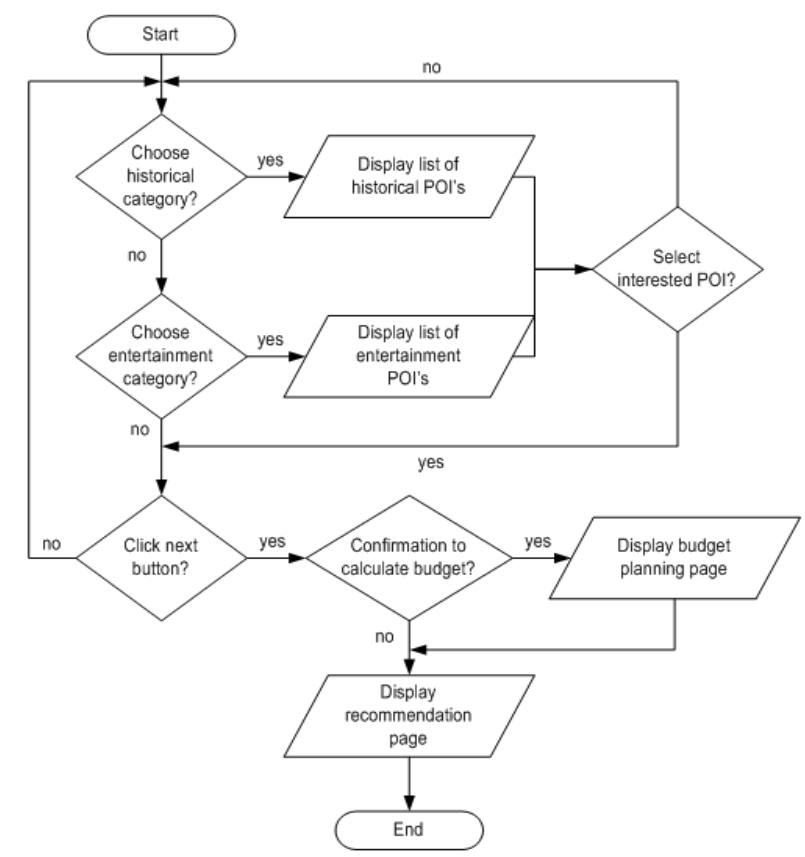

Figure 2. Flowchart diagram for select interested POI 


\subsection{Implementation of Brute Force Algorithm}

We applied the BF algorithm to find the shortest duration to minimize the time taken to visit the desired POI. There were few steps needed to be followed as described by [25] in solving the TSP using BF.

Step 1: Choose a node as the starting point.

Step 2: Look at all weight of the edges that connect with the starting point.

Step 3: List all the possible POI.

Step 4: Calculate the duration of each tour.

Step 5: Choose the shortest tour which is the optimal solution

Step 1: The system will retrieve the traveler's current location as a starting point. Then, the traveler needs to choose a few interesting places. As presented in Figure 3, assume the node A as the current location (starting point), node B as Cheng Ho Cultural Museum, node C as Menara Taming Sari and node D as Melaka Sultanate Palace Museum. The numbers show is the weightage for the duration in minutes.

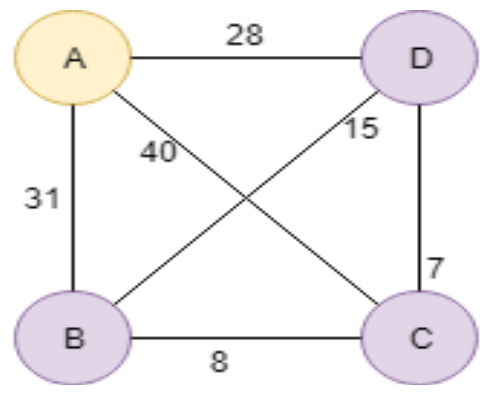

Figure 3. Nodes illustration with duration

Step 2: The number at the route is considered as the weightage which is time duration in hours and minutes. It was given by the Direction API and for this example, minutes is used as the weightage.

Step 3: Possible tours will be listed which are from all nodes, which are A-B-C-D-A, A-B-D-C-A, A-C-B-DA, A-C-D-B-A, A-D-B-C-A, and A-D-C-B-A.

Step 4: Calculating the total duration of each possible tours is listed as in Table 1 using the BF algorithm.

Table 1. Total Duration of Each Possible Tours

\begin{tabular}{cc}
\hline Possible Tours & Duration (minutes) \\
\hline A-B-C-D-A & 74 \\
A-B-D-C-A & 93 \\
A-C-B-D-A & 91 \\
A-C-D-B-A & 93 \\
A-D-B-C-A & 91 \\
A-D-C-B-A & 74 \\
\hline
\end{tabular}

\section{RESULTS AND ANALYSIS}

This section covers the results and findings from the TravelJoy recommendation system. In this study, there were two types of testing conducted which is functionality testing and reliability testing. While reliability testing is tested to prove the results of this project are reliable. It is being categorized into two parts which are the reliability of system versus Google Maps and the reliability of recommendation's result by comparing TravelJoy vs Google Maps and the t-test of the system.

\subsection{Functionality Testing}

The functionality of the system was tested based on the use case testing to make sure the function meets the requirement and works correctly for admin and the traveler. Table 2 shows the result of the functionality test based on use case and Figure 4 shows the snapshot of the main menu TravelJoy recommendation system followed by Figure 5 the budget menu, recommendation of the selected POI sequences and the map. 
Table 2. Functionality Test Result

\begin{tabular}{|c|c|c|c|c|c|}
\hline Component & Test1 & Test2 & Test3 & Test4 & Test5 \\
\hline Sign In & Ok & Ok & Ok & Ok & Ok \\
\hline Add Place of POI & Ok & Ok & Ok & Ok & Ok \\
\hline Update Place of POI & Ok & Ok & Ok & Ok & Ok \\
\hline Delete Place of POI & Ok & Ok & Ok & Ok & Ok \\
\hline Start & Ok & Ok & Ok & Ok & Ok \\
\hline View list of POI & $\mathrm{Ok}$ & Ok & $\mathrm{Ok}$ & Ok & $\mathrm{Ok}$ \\
\hline View details of POI & $\mathrm{Ok}$ & Ok & $\mathrm{Ok}$ & $\mathrm{Ok}$ & $\mathrm{Ok}$ \\
\hline Select & Ok & Ok & Ok & Ok & Ok \\
\hline Choose Interested POI & Ok & Ok & Ok & Ok & Ok \\
\hline Enter planned budget & Ok & Ok & Ok & Ok & Ok \\
\hline Enter number of person on trip & Ok & Ok & Ok & Ok & Ok \\
\hline Calculate & Ok & Ok & Ok & Ok & Ok \\
\hline View recommendation of POI & Ok & Ok & Ok & Ok & Ok \\
\hline View Map & $\mathrm{Ok}$ & Ok & $\mathrm{Ok}$ & $\mathrm{Ok}$ & Ok \\
\hline Exit & Ok & Ok & Ok & $\mathrm{Ok}$ & Ok \\
\hline
\end{tabular}
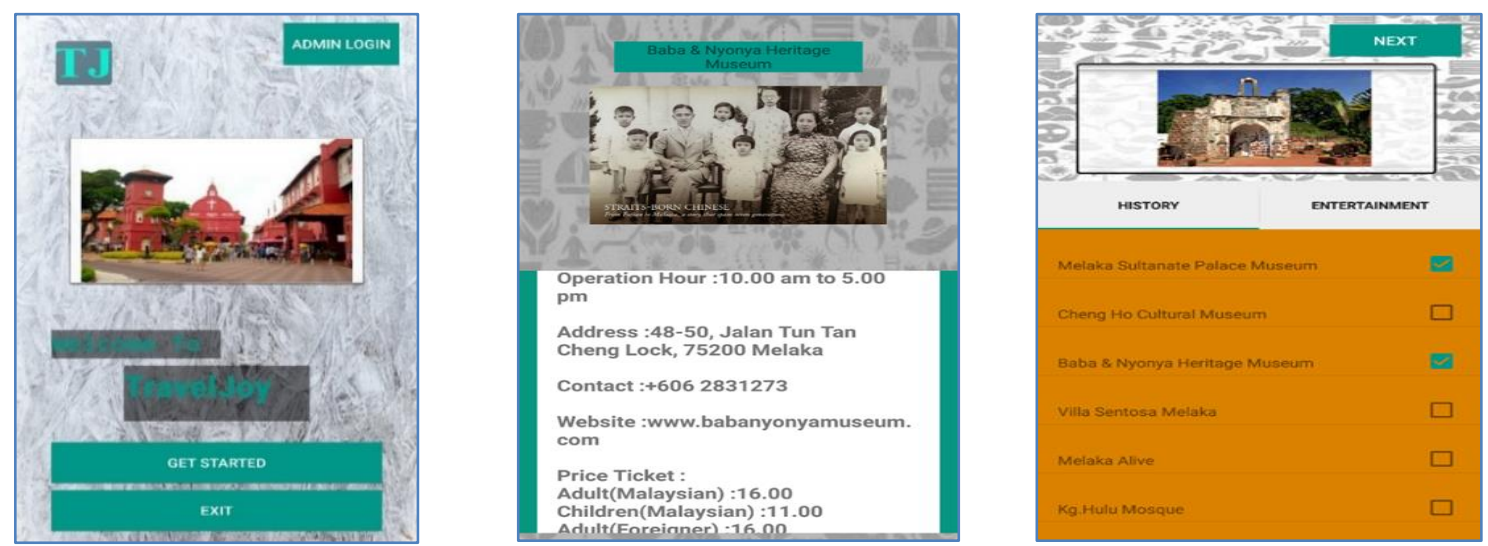

Figure 4. Snapshot of the main menu traveljoy recommendation system
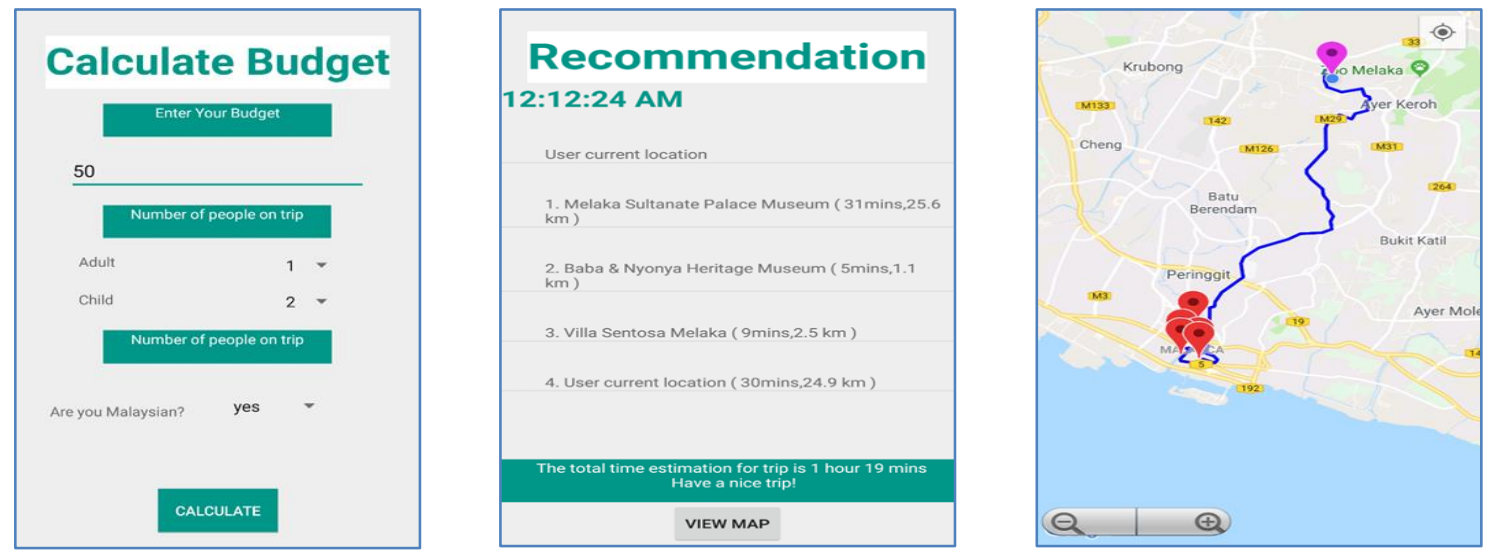

Figure 5. Snapshot of budget calculation and POI squences with map

\subsection{Reliability Testing}

In the reliability testing of the system, we used five selected POIs as identified in Table 3 and two types of reliability are conducted. Firstly, the comparison of duration time taken between TravelJoy and Google Maps and secondly, the validation of TravelJoy recommendation result using manual BF algorithm calculation. 
Table 3. Identification Node for Each Selected Place

\begin{tabular}{cc}
\hline Identification Node & Place Name \\
\hline A & Current Location (McDonald's MITC) \\
B & Baba \& Nyonya Heritage Museum \\
C & Cheng Ho Cultural Museum \\
D & Menara Taming Sari \\
E & Melaka Funland \\
\hline
\end{tabular}

\subsubsection{Reliability Comparison Duration Time Between TravelJoy and Google Maps}

Figure 6 shows the result of duration time taken from node A to node B using TravelJoy recommendation system while Figure 7 shows the result of duration from node A to node B using Google Maps. Based on the same value of 26 minutes from both method, thus, it is proven the reliability of the TravelJoy recommendation system.

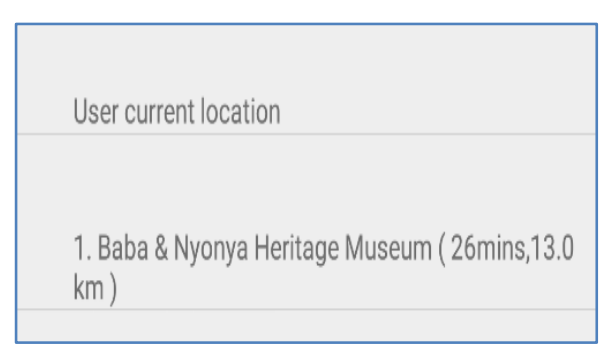

Figure 6. Result from traveljoy

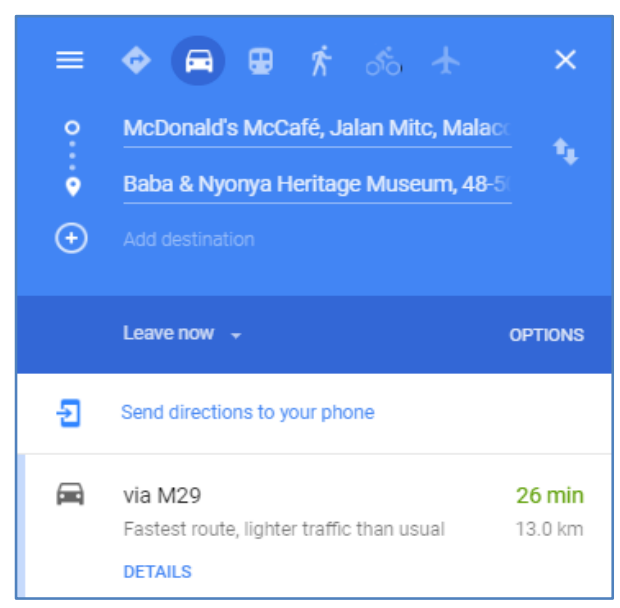

Figure 7. Result from google maps

\subsubsection{Reliability Comparison Duration Time Between TravelJoy and Google Maps}

The second part of reliability testing is the validation of TravelJoy recommendation's result using the same five selected POIs. Based on a manual calculation using the BF algorithm, the result in Table 4, A-B-C-D-E-A (No.1) and A-E-D-C-B-A (No.24) have the smallest total duration which is 65 minutes.

Table 4. List of Possible Tour with Total Duration

\begin{tabular}{cccccc}
\hline No. & Possible Tour & $\begin{array}{c}\text { Total Duration } \\
(\text { minutes })\end{array}$ & No. & Possible Tour & $\begin{array}{c}\text { Total Duration } \\
\text { (minutes) }\end{array}$ \\
\hline 1. & A-B-C-D-E-A & 65 & 13. & A-D-B-C-E-A & 73 \\
2. & A-B-C-E-D-A & 69 & 14. & A-D-B-E-C-A & 80 \\
3. & A-B-D-C-E-A & 75 & 15. & A-D-C-B-E-A & 71 \\
4. & A-B-D-E-C-A & 74 & 16. & A-D-C-E-B-A & 77 \\
5. & A-B-E-C-D-A & 77 & 17. & A-D-E-B-C-A & 70 \\
6. & A-B-E-D-C-A & 72 & 18. & A-D-E-C-B-A & 69 \\
7. & A-C-B-D-E-A & 68 & 19. & A-E-B-C-D-A & 71 \\
8. & A-C-B-E-D-A & 70 & 20. & A-E-B-D-C-A & 76 \\
9. & A-C-D-B-E-A & 76 & 21. & A-E-C-B-D-A & 73 \\
10. & A-C-D-E-B-A & 72 & 22. & A-E-C-D-B-A & 75 \\
11. & A-C-E-B-D-A & 80 & 23. & A-E-D-B-C-A & 68 \\
12. & A-C-E-D-B-A & 74 & 24. & A-E-D-C-B-A & 65 \\
\hline
\end{tabular}

The duration for TravelJoy recommendation shows A-B-C-D-E-A is 1 hour and 7 minutes, or 67 minutes as in Figure 8. It means that from the user current location (McDonald's MITC), the system recommends the user to go Baba \& Nyonya Heritage Museum, followed by Cheng Ho Cultural Museum and Menara Taming Sari, Melaka Funland and finally go back to the user's current location. Despite there was a 
gap of 2 minutes, yet it still considers as shortest duration time taken since the TravelJoy system uses Directed API compared to Google Maps that using GPS.

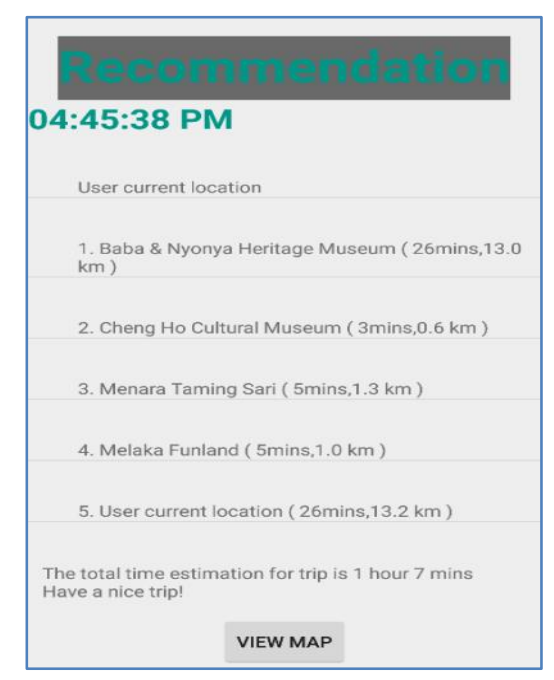

Figure 8. TravelJoy result recommendation

In order to prove the hypothesis that the system is able to minimize the time taken to visit the selected POI, t-test been measured with the $H_{0}$ : There is no significant difference in the experimental time taken using TravelJoy and $H_{1}$ : There is a significant difference in the experimental time taken using TravelJoy. The t-test result as in Table 5 indicates a two-tailed p-value of 0.0079 in which p-value $<0.05$. Therefore, $H_{0}$ is rejected with this sufficient evidence, significant (*p-value $<0.05$ ) and $H_{l}$ is supported as there is a significant difference in the experimental time taken between with and without the TravelJoy recommendation system.

Table 5. Mann Whitney U test for With and Without TravelJoy

\begin{tabular}{|c|c|c|}
\hline \multicolumn{3}{|c|}{ Mann-Whitney U test of Two-Sample } \\
\hline & Without TravelJoy & With TravelJoy \\
\hline Min & 85.000 & 59.000 \\
\hline Max & 121.000 & 81.000 \\
\hline Median & 97.500 & 66.500 \\
\hline Mean & 103.600 & 69.000 \\
\hline $\mathrm{p}$-value & \multirow{2}{*}{\multicolumn{2}{|c|}{$0.00067^{*}$}} \\
\hline & & \\
\hline
\end{tabular}

\section{CONCLUSION}

This study developed the TravelJoy recommendation system using the BF algorithm to calculate the minimum travel time using the TSP approach. The system was tested for functionality and reliability. Both testing provide significant impacts of the recommendation system. TravelJoy provides precise information regarding 20 top places of interest related to entertainment, history and cultural category in Melaka. It is able to help travelers to plan their trip itinerary within the budget during a trip by car and calculate the entrance fees based on total travelers. For future work, it is recommending to allow the traveller to fill up estimation time on how long they would stay at each selected POIs and add it as additional consideration.

\section{REFERENCES}

[1] S. Amir, M. M. Osman, S. Bachok, M. Ibrahim, "Sustaining Local Community Economy Through Tourism: Melaka UNESCO World Heritage City," Procedia Environmental Sciences, vol. 28, pp. 443-452, 2015.

[2] S. Y. Said, N. S. Abdullah, Z. Ithnin, "The Impact of Heritage Tourism to Local Culture and Economy," Pertanika Journal of Social Science And Humanities, vol. 25, pp. 315-322, 2017. 
[3] A. F. Alam, A. C. Er, H. Begum, (2014). "Tourism Development from the Perspectives of Sustainability in Melaka State," in 4th International Conference on Tourism Research (4ICTR), vol. 12, 2014.

[4] A. Smirnov, A. Kashevnik, N. Shilov, N. Teslya, N., A. \& Shabaev, "Mobile Application For Guiding Tourist Activities: Tourist assistant - TAIS," In Conference of Open Innovation Association, FRUCT, pp. 95-100, 2014.

[5] Y. Wang, S. C. F. Chan, G. Ngai, "Applicability of demographic recommender system to tourist attractions: A case study on TripAdvisor, " Proceedings of the 2012 IEEE/WIC/ACM International Conference on Web Intelligence and Intelligent Agent Technology Workshops, WI-IAT 2012, pp. 97-101, 2012.

[6] A. Yahi, A. Chassang, L. Raynaud, H. Duthil, D. Horng, P. Chau, "Aurigo: An Interactive Tour Planner for Personalized Itineraries, "IUI 2015: Proceedings of the 20th International Conference on Intelligent User Interfaces, pp. 275-285, 2015.

[7] K. H. Lim, J. Chan, C. Leckie, S. Karunasekera, "Personalized Tour Recommendation Based on User Interests and Points of Interest Visit Durations, " In IJCAI International Joint Conference on Artificial Intelligence, pp. 17781784, 2015.

[8] L. Jie, Y. Yujiu, L. Wenhuang, "Exploring Personalized Travel Route Using POIs, "International Journal of Computer Theory and Engineering, vol. 7 (2), pp. 126-131, 2015.

[9] A. Aguilera, V. Boutueil, "Urban Mobility and the Smartphone: Transportation, Travel Behavior and Public Policy," Elsevier, 2018.

[10] A. Garcia, P. Vansteenwegen, O. Arbelaitz, W. Souffriau, M. T. Linaza, "Integrating Public Transportation In Personalised Electronic Tourist Guides, "Computers and Operations Research, vol. 40 (3), pp. 758-774, 2013.

[11] E. Hall, Institute for Global Studies Travel Study Handbook, 2015.

[12] W. Husain, L. Y. Dih, "A Framework of A Personalized Location-Based Traveler Recommendation System In Mobile Application, "International Journal of Multimedia and Ubiquitous Engineering, vol. 7 (3), pp. 11-18, 2012.

[13] Z. Yu, S. Zhang, S. Chen, B. Liu, S. Ye, "Research on Traveling Routes Problems Based on Improved Ant Colony Algorithm, " Jinan University, 2013.

[14] C. Beer, E. Gnan, W. U. Birchler, W. U, "Cash on Trial," Vienna: SUERF Conference Proceedings 2016, pp. 5-13, 2016.

[15] J. M. Noguera, M. J. Barranco, R. J. Segura, L. Martínez, "A Mobile 3D-GIS Hybrid Recommender System for Tourism, " University of Ja' en, 2012.

[16] R. Anacleto, L. Figueiredo, A. Almeida, P. Novais, "Mobile Application To Provide Personalized Sightseeing Tours," Journal of Network and Computer Applications, vol. 41 (1), pp. 56-64, 2014.

[17] R. Logesh, V. Subramaniyaswamy, V. Vijayakumar, "A Personalised Travel Recommender System Utilising Social Network Profile And Accurate GPS Data. Electronic Government, an International Journal, vol. 14 (1), pp. 90$113,2018$.

[18] A. Bethapudi, "The Role of ICT in Tourism Industry, " Journal of Applied Economics and Business, vol. 1(4), pp. 67-79, 2013.

[19] I. Brilhante, J. A. Macedo, F. M. Nardini, R. Perego, C. Renso, "Where Shall We Go Today?: Planning Touristic Tours With Tripbuilder," in Proceedings of the 22nd ACM international conference on Information \& Knowledge Management, pp. 757-762, 2013.

[20] S. Bouraga, I. Jureta, S. Faulkner, C. Herssens, "Knowledge-Based Recommendation Systems: A Survey," International Journal of Intelligent Information Technologies (IJIIT), vol. 10(2), pp. 1-19, 2014.

[21] D. Landa-Silva, "Franz Rothlauf: Design of Modern Heuristics," Genetic Programming and Evolvable Machines, vol. 14(1), pp. 119-121, 2013.

[22] M. G. Zaragoza, H. K. Kim, D. S. Han, "Mobile Application Development, Approaches, Advancement and Process," International Journal of Software Engineering and Its Applications, vol.10 (10), pp.79-88, 2016.

[23] V. N. Inukollu, D. D. Keshamon, T. Kang, M. Inukollu, "Factors Influncing Quality of Mobile Apps: Role of Mobile App Development Life Cycle, "International Journal of Software Engineering \& Applications, vol. 5 (5), pp. 15-34, 2014.

[24] A. Kaur, K. Kaur, "Suitability of Existing Software development Life Cycle (SDLC) in context of Mobile Application Development Life Cycle ( MADLC)," International Journal of Computer Applications, vol. 116 (19), pp 1-6, 2015.

[25] Asal 15 A. Sahalot, S. Shrimali, "A Comparative Study of Brute Force Method, Nearest Neighbour And Greedy Algorithms To Solve The Travelling Salesman Problem," International Journal of Research in Engineering \& Technology, vol.2 (6), pp. 59-72, 2014. 